\title{
Passive ranging using an infrared search and track sensor
}

\author{
Maarten de Visser \\ Defence Material Organisation \\ Directorate of Material Royal Netherlands \\ Navy (RNLN) \\ Weapon and Communication Systems \\ v.d Burchlaan 31 \\ NL-2597 PC The Hague, The Netherlands \\ E-mail:m.visser3@mindef.nl
}

\section{Piet B. W. Schwering}

TNO Defence, Security and Safety

Business Unit Observation Systems

P.O. Box 96864

NL-2509 JG The Hague, The Netherlands

\author{
Johannes F. de Groot \\ Thales Naval Systems \\ Business Unit Radar \& Sensors \\ P.O. Box 42 \\ NL-7550 GD Hengelo, The Netherlands
}

\section{Emile A. Hendriks}

Delft University of Technology (TUD)

Faculty of Electrical Engineering

Information and Communication Theory Group

Mekelweg 4

NL-2628 CD Delft, The Netherlands

\begin{abstract}
We present new techniques for passive ranging with a dualband IR search and track (IRST) sensor aboard a ship. Three distance estimation methods are described: the atmospheric propagation model, the apparent surface of the target, and target motion analysis (TMA). These methods are tested on the sensor output of real data during cold water trials (CWTs). They are evaluated by comparing with simultaneously obtained radar reference data at the test site. Results of these three passive ranging and three fusion processes, combining the preceding methods, are presented. This demonstrates the effectiveness of IR passive ranging techniques in the anti-air-warfare scenario. Majority voting fusion shows that improvement of the distance estimation is achieved in the CWT scenario when combining these three different methods. A range-error reduction of $41 \%$ is obtained, and a typical uncertainty of $5 \%$ is at a $8-\mathrm{km}$ distance. During warm water trials (WWTs) the TMA algorithm was adapted to deal with a dynamic environment of the antisurface warfare scenario (ASuW). These WWTs prove that TMA in combination with an IRST system can extend the basic IRST functionality significantly for a dynamic ASuW scenario. (c) 2006 Society of PhotoOptical Instrumentation Engineers. [DOI: 10.1117/1.2173948]
\end{abstract}

Subject terms: passive ranging; infrared system; fusion of passive ranging methods; target motion analysis.

Paper 050328R received Apr. 26, 2005; revised manuscript received Jun. 28, 2005; accepted for publication Jul. 5, 2005; published online Feb. 27, 2006.

\section{Introduction}

Overall situational awareness is of the utmost importance for a command team aboard of a naval vessel. Picture compilation of the surroundings of the ship is done by all the available sensors aboard, with the objectives of knowing where all "players" are and to acquire a positive identification of those units around the naval vessel. By using active sensor systems the naval vessel gives away its position. Consequently, the use of passive systems is necessary. Aboard the Royal Netherlands Navy (RNLN) Air Defense Command Frigates (ADCF) are several passive sensor systems are available, an one of them will be a dual-band IR search and track (IRST) system. The IRST system constantly scans the environment of the ship and automatically generates 2-D tracks of target objects. The missing parameter is the distance estimation to the target. By applying different passive ranging methods it should be possible to passively obtain 3-D data of a target, which increases the situational and tactical awareness of the command team aboard the naval vessel and enables them (when the accuracy of the distance estimate is high enough) to use a pas-

0091-3286/2006/\$22.00 @ 2006 SPIE sive sensor as a fire control sensor. There is currently no system available that acquires 3-D information without actively transmitting energy.

This research focuses on a dual-band IRST system. There are several reasons why the dual-band IRST system is placed aboard the ADCF. The IRST system contributes to the ship multisensor database, and its contribution is particularly vital in the following conditions:

1. during ship emission restrictions

2. when active sensor(s) performance is degraded by electronic countermeasures

3. when active sensor(s) performance is degraded by multipath interference

4. when the environment favors infrared propagation over radar propagation

Despite all these contributions, there is one disadvantage: the current passive sensor to be placed aboard the ADCF is not able to obtain 3-D tracks that include range.

In this paper, we present our study of the feasibility of obtaining range information from passive IR sensors only, with sufficiently high accuracy, in particular in the anti-airwarfare (AAW) scenario. The study consists of the applica- 


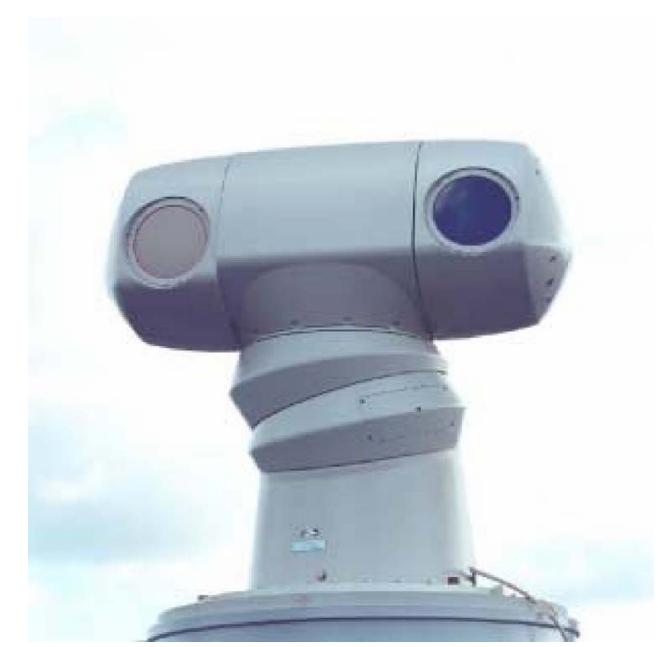

Fig. 1 Dual-band stabilized sensor head of the long-range IRST system. The system rotates at $1 \mathrm{~Hz}$ and has elevation coverage optimized for sea skimming missiles and surface contacts. The platform is stabilized over three axes. The sensor at the left side, as seen from the back, is the long-wavelength (LW) $(8.0=$ to 10.0 $-\mu \mathrm{m})$ sensor and the medium-wavelength (MW) $(3.0$ to $5.0-\mu \mathrm{m})$ sensor is at the right.

tion, comparison, and combination of different ranging algorithms. We limit ourselves to the following four boundary conditions:

1. The output of the sensor for the distance estimation consists of 2-D track information (bearing and elevation) and bearing and elevation rate.

2. The images belonging to the track information of the IR cameras are available.

3. Real-time aspects of processing, uncertainty not directly linked to the distance estimation and design aspects of the used IRST system, fall beyond the scope of this paper.

4. Meteorological and scenario data is available-in real time.

The IRST (Ref. 1) consists of two IR sensors, processing equipment, and several control and supply units. Both sensors are placed in a stabilized sensor head that is shown in Fig. 1.

The sensor will be placed aboard the Netherlands ADCF. Its main purpose is to detect and track incoming sub- and supersonic sea-skimming missiles. Before actual integration of the sensor in the combat management system of the ship, a preproduction model is designed by the manufacturer and placed and evaluated at a land-based test site (LBTS) and a sea-based test site (SBTS). The system is submitted to cold water trials (CWTs) and warm water trials (WWTs) at, respectively, the LBTS and the SBTS.

The paper is organized as follows. Section 2 summarizes related work and discusses the most appropriate methods for passive ranging with the IRST system. Section 3 continues with the combined method for passive ranging used for evaluating the CWT scenario. Section 4 gives the experimental results. Results of a dynamic scenario during WWTs are also presented. Section 5 is the discussion and in Sec. 6, the conclusions are formulated.

\section{Passive Ranging}

\subsection{Related Work}

Before discussing our research in the next sections, an overview of related papers is presented here. Related work on passive ranging is reported by a considerable number of authors. These references describe a variety of possible passive ranging techniques, applications and combinations of different sensor systems. A summary is shown in Table 1 .

Table 1 Comparison of related work.

\begin{tabular}{|c|c|c|c|c|}
\hline Author & Sensor & Method & Fusion & Experiments \\
\hline Blackman and Popoli ${ }^{2}$ & IRST & $\begin{array}{l}\text { S, IDD, TMA, MS, } \\
\text { AS }\end{array}$ & Yes & Real and simulated \\
\hline Van Sweeden et al. ${ }^{3}$ & IRST & S, IDD, APM, AS & Yes & None \\
\hline de Groot $^{4}$ & IRST & Interactive TMA & No & Real and simulated \\
\hline Kemp ${ }^{5}$ & IRST & AS & No & $\mathrm{N} / \mathrm{A}$ \\
\hline Schwering et al. ${ }^{6}$ & Staring IRST & APM & No & None \\
\hline Aytac and Barshan ${ }^{7}$ & Infrared & Intensity, 2 sensors & Yes & Real and simulated \\
\hline Sasaki et al. ${ }^{8}$ & CCD camera & Intensity, 2 sensors & No & Real \\
\hline Ono and Komatsu ${ }^{9}$ & PCC & Intensity & No & Real and simulated \\
\hline Ruben and Michalowicz ${ }^{10}$ & IRST & TMA & No & Simulated \\
\hline
\end{tabular}

$\overline{\text { An N/A denotes that the information cannot be deduced form the reference. Abbreviations used are S, }}$ stereo imaging; IDD, intensity distance diagram; TMA, target motion analysis; MS, multispectral analysis; AS, apparent surface of the target; APM, atmospheric propagation model; and PCC, photoncounting camera. 
Techniques mentioned for passive ranging are stereo imaging (S), use of intensity-distance diagrams (IDD), target motion analysis (TMA), multispectral analysis (MS), calculating the apparent surface of a target (AS), using atmospheric propagation models (APMs), combining a priori knowledge from an active sensor such as a laser range finder, and using a dual-platform combination for sensor fusion. An important conclusion from the references is that little research is done for single-operating long-range IR sensors. An actual implementation and evaluation for an actual existing IRST system with live target data has not been found. Furthermore, the combination of different passive ranging techniques is, to the best of our knowledge, not practically implemented in an IRST system.

Based on the methods described in the related work of Table 1 (S, IDD, TMA, MS, AS, APM) and the output of the IRST sensor, TMA, APM, and AS are further investigated. Stereo imaging $(\mathrm{S})$ is not a good candidate because the instantaneous field of view of the sensors limits the use of this method for passive ranging to a distance of about $2 \mathrm{~km}$. The IDD and MS methods are not taken into account because of unavailable information.

\subsection{Methods for Distance Estimation}

The output of the IRST sensor consists of bearing and bearing rate, elevation and elevation rate of the target, and the images of both camera systems. This information is delivered in successive time steps. The three selected methods are described in the next subsections. A comparison of the methods concludes in Sec. 2.2.

\subsubsection{APMs}

Based on the altitude of the target and more scenario information such as meteorological data, it is possible to make a distance estimate when the target is observed for the first time. There are several distortions to deal with at IR frequencies when calculating the distance to the horizon or an object at the horizon. ${ }^{11-14}$ There are effects that cause absorption and the effective earth radius will change because of refraction. Furthermore scintillation [rapid fluctuations in signal amplitude (fading rate per second) due to atmospheric effects] also affects the propagation of IR rays. These effects are not always a disadvantage for the distance performance of IR sensors. Certain atmospheric conditions enlarge the distance of first detection of an object in the IR spectrum.

Various models are available to estimate the propagation of IR rays in the trajectory of interest. Meteorological input data is needed and of great importance for the accuracy of the used model. ${ }^{14}$ Some models are

1. MODTRAN, a computer model for prediction of the optical properties of the atmosphere

2. IRTool, a model for prediction of the effect of refraction

3. ARTEAM, a ray-tracing model for electro-optic (EO) applications

4. IRBLEM, an IR boundary layer effects model ${ }^{15}$

Since IRBLEM is available for the RNLN and the model is an improved version of MODTRAN for usage in a maritime environment, we use this model.
The IRBLEM estimates the influence of absorption, refraction, and scintillation on the propagation of IR waves in the so-called "marine boundary layer" above the sea surface. Previous research on this subject ${ }^{11-14}$ shows that this method provides realistic first-distance estimates. Besides a first-distance estimate, the model IRBLEM gives the effective transmission for the propagation path. Furthermore, the target intensity is approximated from the IR images by the use of a calibration equation:

$I=\frac{\sum\left(I_{\text {peak_target }}-I_{\text {background }}\right) \delta \Omega}{\sigma_{\text {background }}} \mathrm{NEI}_{\mathrm{LW}_{-} \text {band }}$,

where $I$ is the intensity, $I_{\text {peak_target }}$ is the peak intensity of the target, $I_{\text {background }}$ is the mean intensity of the background, $\delta \Omega$ is the solid space angle of the irradiance field of view (IFOV), $\sigma_{\text {background }}$ is the standard deviation of the empty sky background, and $\mathrm{NEI}_{\mathrm{LW} \text { band }}$ is the estimated noise equivalent irradiance of the LW camera. During CWTs the LW band dominated the MW band, which complicates the possible combination of both bands. We choose to use the dominant spectral band. Because an object emits radiation all around and we receive the intensity at a distance $R$, the intensity $(I)$ is ${ }^{16}$

$I=W \frac{\tau}{R^{2}}$,

where $W$ is the target radiant intensity, and $\tau$ is the transmission to the distance $R$. The radiant intensity of the target is assumed known, but estimated here from the TMA process.

\subsubsection{AS of the target}

Processing and interpretation of the recorded images adds information to the solution of the distance estimation problem. The optical AS of the target changes when distance is changing. The region of interest (i.e., the incoming target) will grow while approaching the ship (larger apparent surface). Figure 2 shows some images taken by the sensor.

The AS method requires an extended target (a target that extends over multiple pixels) imaged at different distances, with the distance difference known. ${ }^{3}$ The first detection of an incoming target will display a tiny spot in the image. A gray-value-intensity-based threshold procedure is used to determine the size of the target. The IRST sensor first detects the target. Second, examination of the boundary regions in the image around the target of interest is performed to obtain a threshold value. Finally, computation of the surface of the target of interest is done.

The area of an extended target may also change with a changing viewing aspect, but if we assume a straight incoming target, then

$A=\frac{c}{R^{2}}$,

where $A$ is the number of pixels of the target, $c$ is the constant of proportionality, and $1 / R^{2}$ represents the influence of distance on the target area. From this it follows that 

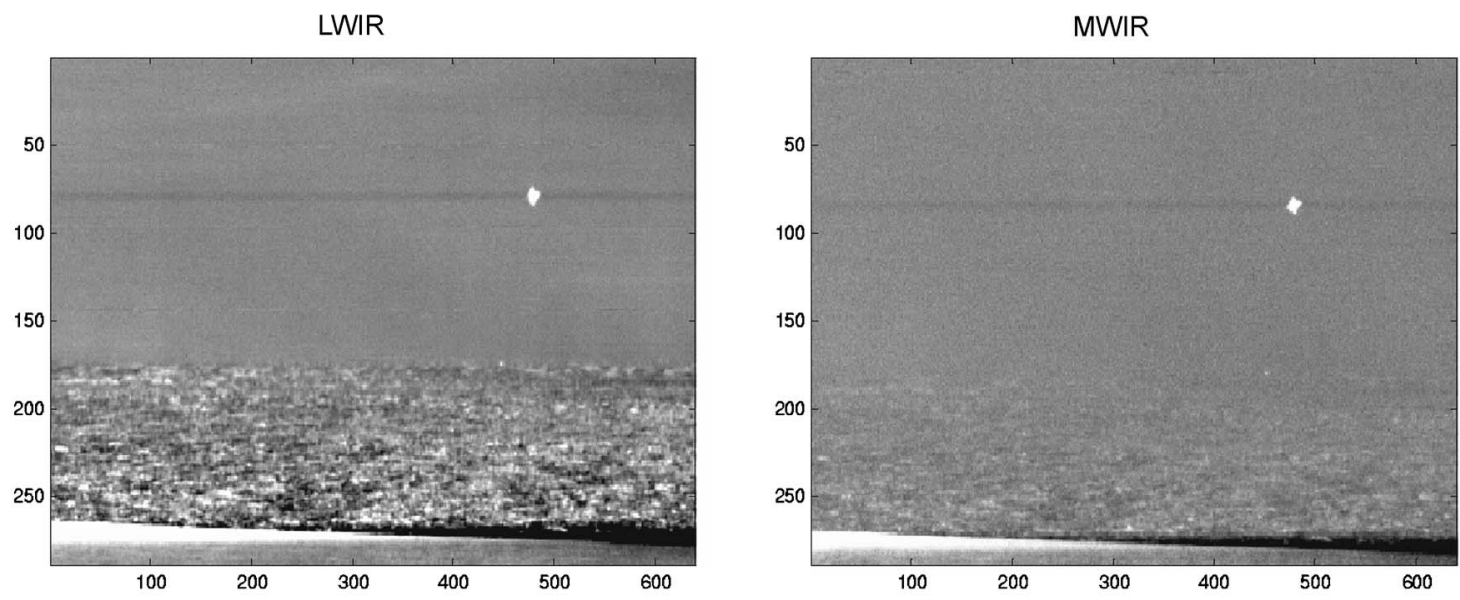

Fig. 2 IR images from an airplane (left is LWIR, right is MWIR); the axes are pixel numbers; and the mean has been removed for each line.

$\frac{\mathrm{d} A}{\mathrm{~d} R}=-2 \frac{c}{R^{3}}$.

Combining Eqs. (3) and (4) yields

$R=-2 A \frac{1}{(\mathrm{~d} A / \mathrm{d} R)}$.

When $\mathrm{d} A / \mathrm{d} R$ is approximated by $\Delta A / \Delta R$, representing the ratio of the measured changes in target area and distance respectively, an estimate of the distance $R$ is obtained. A limitation of this method is that an initial reference distance and the changes in distance are required. Another limitation is that this method is only useful for extended targets.

\subsubsection{TMA}

A distance estimation method based on angle measurements is TMA. Using target tracking based on bearing information created by own-ship movement, the distance is estimated. Because of the very accurate bearing measurements of IR sensor systems, TMA looks very suitable to use for distance estimation with an IRST sensor. There are several variations of TMA; single-leg Ekelund ${ }^{17}$ for targets with bearing rate, cross measurements for targets with no or small own velocity, or the usage of TMA-based filters ${ }^{18}$ for dynamic targets in operational scenarios. The TMA principles used during our tests are described in the following.

TMA used principle for dynamic targets. The TMA principle during WWTs is based on batch-type filters. Those types of filters have been used in passive sonar tracking systems. This batch-type filter is very suitable in a passive tracking system, especially in an optronic system, where angle measurements are very accurate. Due to the lack of range measurements in a passive system, the complete state vector of the target cannot be resolved. However, if the sensor is moving with a nonconstant course or speed, the complete target state vector is automatically resolved. The requirement is that the sensor position with respect to an inert reference point is known at any time with sufficient accuracy. The batch filter is very suitable for adding a pri- ori knowledge about the target dynamic constraints or other sensor measurements to the batch. Also passive measurements from other sensors of other platforms could be added.

To be able to estimate the state of the target, an assumption must be made about the target dynamic model. Next we illustrate a constant velocity target model. This model has 6 degrees of freedom. Other target models, such as constant height and fixed position, reduce the degrees of freedom.

Figure 3 visualizes the geometry of the dynamic TMA process is visualized. Here $\overline{p_{\text {sensor }}}(t)$ is the 3-D Cartesian position of the IRST sensor with respect to a fixed reference point $O, \overline{p_{t}}(t)$ is the position of the target at time $t$ with respect to the fixed reference point $O, \overline{p_{S}}(t)$ is the sensor relative (measured) position of the target at time $t$, and $\overline{\mathbf{v}}_{t}(t)$ is the speed vector of the target. Suppose our sensor position is known at every time $t$ with respect to an inertial reference point $O$. The measured bearing $B(t)$ and elevation $E(t)$ (not given in 2-D Fig. 3, this is the measured elevation angle of the target) are given by

$B(t)=\arctan \left[x_{s}(t) / y_{s}(t)\right]$

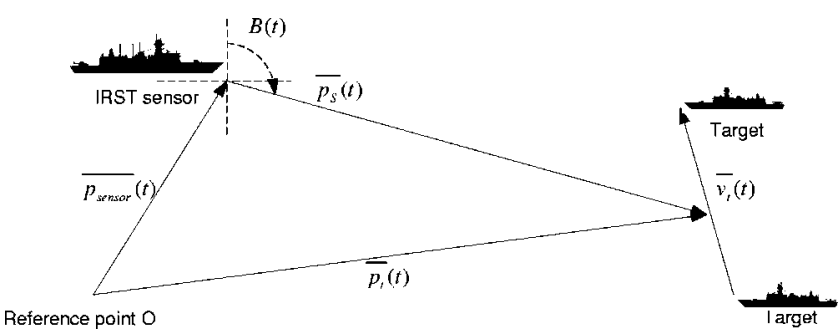

Fig. 3 Visualization of dynamic TMA vectors and geometry. 
$E(t)=\arcsin \left[\frac{z_{s}(t)}{\left|p_{s}(t)\right|}\right]$,

where

$\overline{p_{s}}(t)=\left[\begin{array}{l}x_{s}(t) \\ y_{s}(t) \\ z_{s}(t)\end{array}\right]=\overline{p_{t}}(t)-\overline{p_{\text {sensor }}}(t)$,

and $x_{s}(t), y_{s}(t)$, and $z_{s}(t)$ are the components of state vector of the target. The model equation at $t_{0}$ looks like:

$\mathbf{H}=\left[\begin{array}{cccccc}1 & 0 & 0 & \left(t-t_{0}\right) & 0 & 0 \\ 0 & 1 & 0 & 0 & \left(t-t_{0}\right) & 0 \\ 0 & 0 & 1 & 0 & 0 & \left(t-t_{0}\right)\end{array}\right]$,

where $\mathbf{H}$ is the state model and $t$ and $t_{0}$ are the time and the time at $t=0$. Then,

$$
\begin{aligned}
\mathbf{H}\left[\begin{array}{l}
\overline{p_{t}}\left(t_{0}\right) \\
\overline{v_{t}}\left(t_{0}\right)
\end{array}\right]= & \overline{p_{\text {sensor }}}(t)+\overline{p_{s}}(t)=\overline{p_{\text {sensor }}}(t)+R(t) \\
& \times\left[\begin{array}{c}
\sin B(t) \cos E(t) \\
\cos B(t) \cos E(t) \\
\sin E(t)
\end{array}\right],
\end{aligned}
$$

where $R(t)$ is the range at time $t$. Eliminating the range equation, by using the following transformation:

$\mathbf{L}_{t}=\left[\begin{array}{ccc}\cos B(t) & -\sin B(t) & 0 \\ -\sin B(t) \sin E(t) & -\cos B(t) \sin E(t) & \cos E(t)\end{array}\right]$

results in

$\mathbf{L}_{t} \mathbf{H}\left[\begin{array}{c}\overline{p_{t}} \\ \overline{v_{t}}\left(t_{0}\right)\end{array}\right]=\mathbf{L}_{t} \overline{p_{\text {sensor }}}(t)$.

This is the basic equation for each observation. For every measurement $B, E$ of a track at $t=t_{i}$ we can add the basic equation to an overdetermined set of equations, which can be resolved by using singular value decomposition (SVD):

$\mathbf{A} \overline{\mathbf{x}}=\overline{\mathbf{b}}$

$\mathrm{USV}^{T} \overline{\mathbf{x}}=\overline{\mathbf{b}}$,

$\overline{\mathbf{x}}=\mathrm{VS}^{-1} \mathbf{U}^{T} \overline{\mathbf{b}}$.

If the set is nonsingular the state vector $\overline{\mathbf{x}}$ can be resolved directly, otherwise the singular diagonal elements in $\mathbf{S}^{-1}$ can be replaced by variables. This will result in a solution space, where the real solution can be written as a linear combination of range and null space vectors. With a singular set of equations some observable states can be derived such as time to go (TTG), time, and direction of the closest point of approach (CPA).

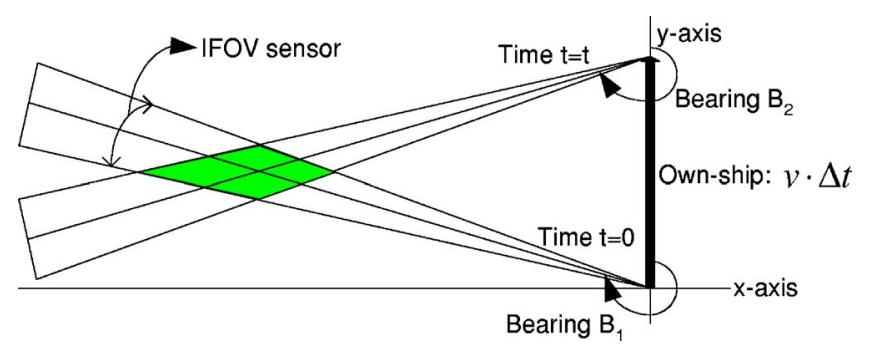

Fig. 4 Geometrical visualization of the static TMA bearings.

TMA used principle for stationary targets. The geometrical layout of the static TMA process is visualized in Fig. 4. A limitation is that the ship must move over time to collect different bearing information. Movement of the ship is indicated by the thick black arrow to the north $(\nu \Delta t)$. For the bearing at time $t=0$ the following equations apply:

$x=R \sin \left(B_{1}\right)$

and

$y=R \cos \left(B_{1}\right)$,

where $x$ is the $x$ coordinate of the target, $y$ is the $y$ coordinate of the target, $R$ is the range of the target, and $B_{1}$ is the bearing from the own ship to the target at time $t=0$. Combining Eqs. (14) and (15) gives

$y=\left[1 / \tan \left(B_{1}\right)\right] x$.

At time $t=t$, Eq. (16) becomes

$y=v \Delta t+\left[1 / \tan \left(B_{2}\right)\right] x$,

where $v$ is the speed of the own ship, $\Delta t$ is the time between the bearing measurements and $B_{2}$ is the bearing at time $t=t$. Combining Eqs. (16) and (17) gives the intersection point $(x, y)$. The range equation is

$$
\begin{aligned}
R= & \left(x^{2}+y^{2}\right)^{1 / 2}\left(\left[\frac{v \Delta t}{1 / \tan \left(B_{1}\right)-1 / \tan \left(B_{2}\right)}\right]^{2}\right. \\
& \left.+\left\{\left[1 / \tan \left(B_{1}\right)\right] \frac{v \Delta t}{1 / \tan \left(B_{1}\right)-1 / \tan \left(B_{2}\right)}\right\}^{2}\right)^{1 / 2} .
\end{aligned}
$$

Equation (18) is simplified to

$R=\frac{-v \Delta t \sin \left(B_{2}\right)}{\sin \left(B_{1}-B_{2}\right)}$

The most relevant assumptions with this TMA analysis are (1) the target is nonmoving or travels in a straight line with a constant velocity and (2) the availability of a minimum of two sets of track data of the target.

A limitation of this method is that the greater the velocity of the target in relation to the velocity of the own plat- 
Table 2 Advantages and disadvantages per method.

\begin{tabular}{|c|c|c|c|}
\hline Method & Advantage & Disadvantage & Range Equation \\
\hline APM & $\begin{array}{l}\text { Additional scenario } \\
\text { information available from } \\
\text { atmospheric model }\end{array}$ & $\begin{array}{l}\text { Meteorological information needed. } \\
\text { Many input parameters } \\
\text { Target radiant intensity needed } \\
\text { Indicative first distance estimate and velocity of target } \\
\text { needed }\end{array}$ & $R=\underset{11}{\left(W \frac{\tau}{I}\right)}$ \\
\hline AS & $\begin{array}{l}\text { Moving target indication } \\
\text { available }\end{array}$ & $\begin{array}{l}\text { Initial reference distance needed. } \\
\text { Changes in distance needed } \\
\text { Useful for extended targets }\end{array}$ & $R=-2 A \frac{1}{\mathrm{~d} A / \mathrm{d} R}$ \\
\hline TMA (stationary) & $\begin{array}{l}\text { Only bearing and own } \\
\text { ship information needed }\end{array}$ & $\begin{array}{l}\text { Inaccurate if velocity target is larger than own ship } \\
\text { Ship movement required }\end{array}$ & $R=\frac{-v \Delta t \sin \left(B_{2}\right)}{\sin \left(B_{1}-B_{2}\right)}$ \\
\hline TMA (dynamic) & $\begin{array}{l}\text { Resolving 3-D position } \\
\text { and velocity of the target; } \\
\text { suitable for adding a priori } \\
\text { knowledge and other } \\
\text { sensor measurements }\end{array}$ & $\begin{array}{l}\text { Own ships maneuvers, or a priori } \\
\text { knowledge about target dynamics } \\
\text { required; suitable for slow moving air } \\
\text { or surface targets at relative short } \\
\text { range (less than } 10 \mathrm{~km} \text { ) }\end{array}$ & $\begin{array}{l}\text { Dependent on the } \\
\text { geometry of the tracking } \\
\text { problem }\end{array}$ \\
\hline
\end{tabular}

form, the worse will be the distance estimation. With this TMA method it is possible to estimate the distance when the target comes straight toward the ship.

\subsubsection{Comparison of methods}

A comparison between the three methods is given in Table 2. The distance estimates of the methods APM, AS of the target, and TMA (stationary) are implemented separately as well as combined in the CWT sections. The TMA method has a direct numerical output for the distance estimation, and the APM and AS methods require an initial reference. This reference distance estimation is given by the TMA method.

\section{Combined Method Used in CWT Scenario}

Under the assumption that all methods described in Sec. 2 provide useful, but not ideal, range data, we consider the possibility of combining the different range estimates to improve the range result. The distance estimation of the specific methods is then input for a fusion process. The fusion itself is based at decision levels, so decision level fusion is chosen (in Ref. 19 a distinction is made between data-, feature-, and decision-level fusion). As methods for fusion we choose "best method," "voting," and "weighted average."

\subsection{Fusion Scheme}

An important factor for information fusion is that the output of each method must give a confidence level that describes the (un)certainty. A confidence level expresses a confidence or belief in the uncertainty of a distance estimate at a certain time, which is expressed as a number between 0 and 1 . The confidence values are used to indicate an order in the uncertainty of the methods. This means that a higher confidence value implies a lower uncertainty number for a method. Figure 5 presents the layout for the fusion of the distance estimations.

The decision-level information fusion method adjusts the weight of each method by means of a mapping (weighting) function. This mapping function requires one parameter $\left(u_{i}\right.$, dependent on the confidence level) for each method $i$ and adjusts differences between the three methods. The mapped distance estimation methods are combined with a decision-level fusion function to acquire a single distance estimation. $^{19,20}$

\subsection{Uncertainty in the Methods and Confidence Levels}

Uncertainty in the three chosen methods is present because of parameter predictions, inaccurate measurements, and/or dependency on other methods. We implemented uncertainty in all input parameters for the different range equations, and the input parameters are assumed independent.

\subsubsection{APM}

Uncertainty in the APM method is incorporated by adding an uncertainty percentage to all input parameters in the range Eq. (2). The uncertainty in the atmospheric input pa-

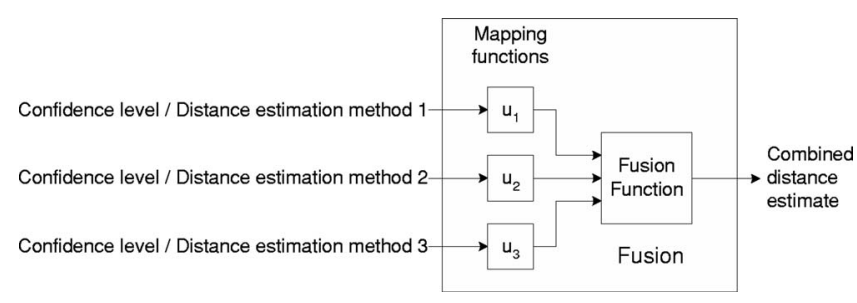

Fig. 5 Information fusion flow diagram. 
rameters of the IRBLEM gives an overall uncertainty in the transmission $\tau$. First, the sensitivity of the transmission for the different input parameters is determined (atmospheric input parameters like solar radiation, wind speed, etc. have negligible influence on the total transmission). Then four remaining input parameters for determining the transmission are combined in one uncertainty number for the transmission:

$\tau=\tau_{\text {irblem }}(p$, air_temp, rel_hum, water_temp $)+\Delta \tau$,

where $\tau$ is the transmission with uncertainty; $\tau_{\text {irblem }}$ is the transmission without uncertainty dependent of the pressure $(p)$, the air temperature (air_temp), the relative humidity (rel_hum) and the water temperature (water_temp); $\Delta \tau$ is the uncertainty in the transmission $\tau$. The uncertainty is calculated by:

$$
\begin{aligned}
\Delta \tau= & {\left[\left(\Delta \tau_{p}\right)^{2}+\left(\Delta \tau_{\text {air_temp }}\right)^{2}+\left(\Delta \tau_{\text {rel_hum }}\right)^{2}\right.} \\
& \left.+\left(\Delta \tau_{\text {water_temp }}\right)^{2}\right]^{1 / 2}
\end{aligned}
$$

where $\Delta \tau_{p}$ is the uncertainty in the transmission $\tau$ when only the uncertainty in the pressure is incorporated. We applied a similarly method for the uncertainties in air temperature, relative humidity, and water temperature. The uncertainty in the pressure is calculated by

$\Delta \tau_{p}=\left(\left|\tau_{p_{-} \min }-\tau_{\text {irblem }}\right|+\left|\tau_{p_{-} \max }-\tau_{\text {irblem }}\right|\right) / 2$,

where $\tau_{p_{\text {_min }}}$ is the transmission calculated with the model for the minimum pressure and $\tau_{p_{\text {_max }}}$ is the transmission with the maximum pressure. Analogous to Eq. (22), the uncertainty for the other three atmospheric input parameters is determined. Analogously to the uncertainty calculations for the transmission, the uncertainty for the intensity $I$ in Eq. (1) is calculated with the appropriate input parameters. The uncertainty in the target radiant intensity input parameter $W$ in Eq. (2) is estimated by calculating this parameter with the information from the TMA method. Here it is determined from the TMA process to be 1460 to $1650 \mathrm{~W} / \mathrm{sr}$. This estimate of the parameter $W$ is calculated with Eq. (2), where $I\left(=11.31 \mathrm{~W} / \mathrm{m}^{2}\right)$ is derived from the available images, an estimate of $R(=7.9$ to $8.4 \mathrm{~km})$ is available from the TMA (stationary) process, and $\tau(=0.49)$ is available from the IRBLEM model. An estimate of the parameter $W$ should be known for all available targets.

The total uncertainty in the range $\Delta R$ in Eq. (2) is calculated for the method APM as follows:

$\Delta R=\left[(\Delta \tau)^{2}+(\Delta W)^{2}+(\Delta I)^{2}\right]^{1 / 2}$,

where $\Delta \tau, \Delta W$, and $\Delta I$ are the impacts in the range uncertainty of all the uncertainties in the range estimate due to transmission, intrinsic target radiation, and image intensity, respectively. After calculation of the derivatives of range Eq. (2), we get:

$\frac{\Delta R}{R}=\left[\left(\frac{\Delta \tau}{2 \tau}\right)^{2}+\left(\frac{\Delta W}{2 W}\right)^{2}+\left(\frac{\Delta I}{2 I}\right)^{2}\right]^{1 / 2}$.

This means that the total uncertainty in the APM method is dependent on the chosen uncertainty percentages in the transmission, target radiant intensity, and the intensity. We define the confidence level (CL) of the method APM as 1 minus the uncertainty in range of the method divided by the range:

$\mathrm{CL}=1-\frac{\Delta R}{R}$

\subsubsection{AS}

Uncertainty in the AS method is incorporated by adding an uncertainty percentage to all input parameters in the range of Eq. (5). The approach of the uncertainty calculations, as well as for the CL calculations, is the same as for the APM method. The total uncertainty in the range $\Delta R$ in Eq. (5) for the AS method and the calculation of the derivatives give:

$\frac{\Delta R}{R}=\left[\left(\frac{\Delta A}{A}\right)^{2}+\left(\frac{\Delta \mathrm{d} A}{\mathrm{~d} A}\right)^{2}+\left(\frac{\Delta \mathrm{d} R}{\mathrm{~d} R}\right)^{2}\right]^{1 / 2}$.

\subsubsection{TMA for stationary targets}

Uncertainty in the TMA method is incorporated by adding an uncertainty percentage to the input parameters in the range of Eq. (19). After applying analogous equations [e.g., Eqs. (20)-(23)] for the TMA method, the uncertainty in the range estimated by the method TMA is given by

$$
\begin{aligned}
\frac{\Delta R}{R}= & \left(\left[-\frac{\Delta B_{1}}{1 / 2 \sin \left(2 B_{1}-2 B_{2}\right)}\right]^{2}\right. \\
& +\left(\frac{\Delta B_{2}\left\{-2\left[\cos \left(B_{2}-2 B_{1}\right)-\cos B_{2}\right]\right\}}{\left[2 \sin B_{2}-\sin \left(3 B_{2}-2 B_{1}\right)+\sin \left(B_{2}-2 B_{1}\right)\right]}\right)^{2} \\
& \left.+\left(\frac{\Delta v}{v}\right)^{2}+\left[\frac{\Delta(\Delta t)}{\Delta t}\right]^{2}\right)^{1 / 2} .
\end{aligned}
$$

Based on the preceding uncertainty analysis for the different methods the confidence levels of the methods are established [according to Eq. (25)].

\subsection{Methods Used for Fusion}

The decision fusion methods "best," "weighted average," and "majority voting" were chosen to use in the CWT scenario. This choice was made because these "simple" methods already give satisfying results.

\subsubsection{Best method}

The best method is probably the simplest form of fusion. The best method just selects the best distance estimation method available at that time. This method is based on the best (highest) CL per time step. No mapping function is used. "Selection" is the fusion function used.

\subsubsection{Weighted average method}

This method is stated as follows:

$R_{\text {combined }}=u_{\mathrm{APM}} R_{\mathrm{APM}}+u_{\mathrm{AS}} R_{\mathrm{AS}}+u_{\mathrm{TMA}} R_{\mathrm{TMA}}$,

where $u_{i}$ is the adjusted mapping functions $\left(u_{i}\right.$ $=\mathrm{CL}_{i} / \Sigma \mathrm{CL}_{i}$ ), the average $\mathrm{CL}_{i}$ (on the history) per method is confidence levels, and $R_{i}$ is the distance estimate for a 


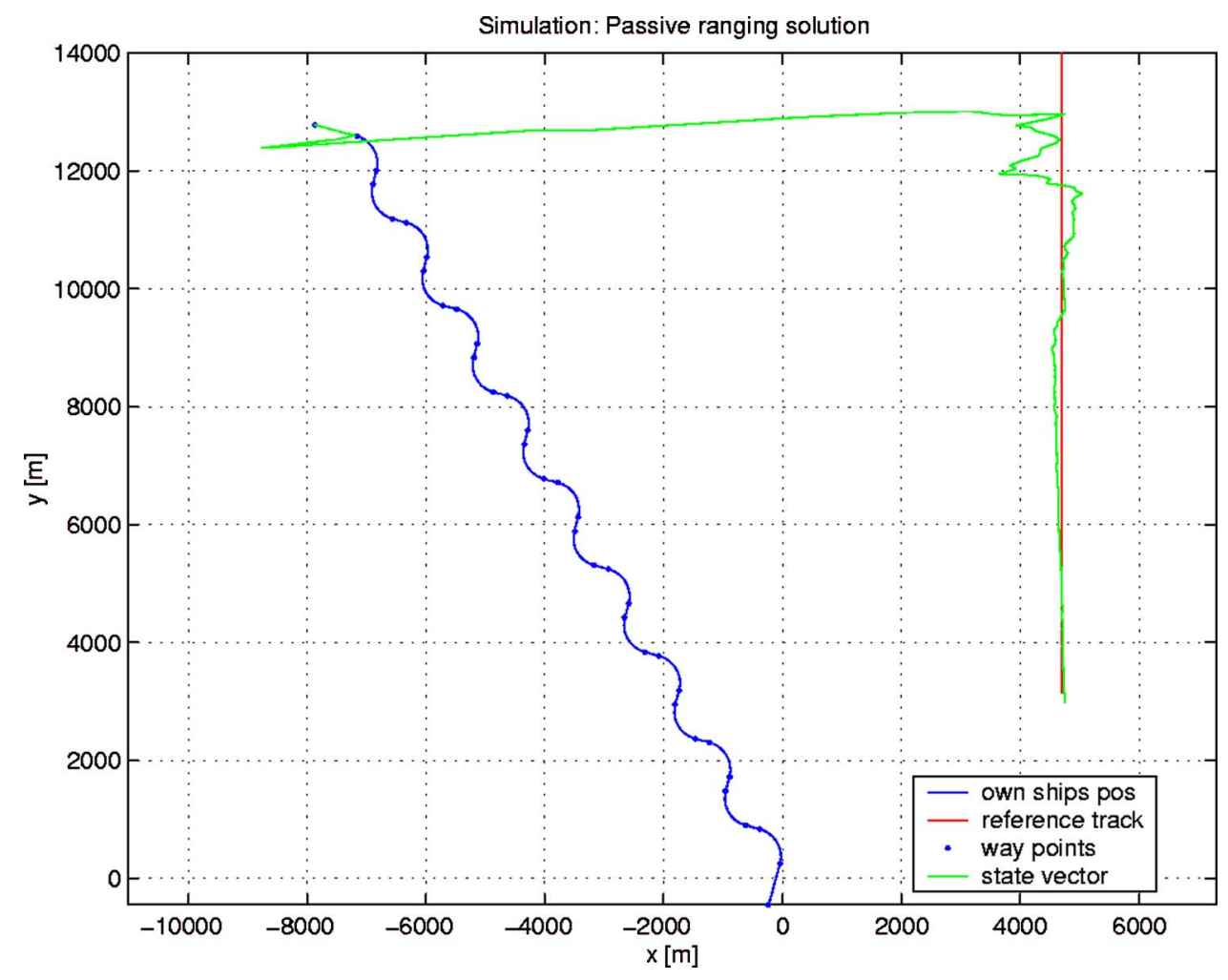

Fig. 6 Surface geometry of an example of a WWT scenario with blue as the own ship track, red as the target reference data, and green as the TMA state vector.

specific method (index $i$ ). Its fusion function is "summation."

\subsubsection{Majority voting method}

Voting fusion is described by thresholds. A vote is given if the method has a confidence level that is higher than a threshold. The votes are summed and a second threshold selects between one out of three ("or" voting), 2 out of 3 (majority voting), or 3 out of 3 ("and" voting) votes. The voting fusion function and the mapping are given by

$$
\begin{aligned}
F\left(\mathrm{CL}_{\mathrm{APM}}, \mathrm{CL}_{\mathrm{AS}}, \mathrm{CL}_{\mathrm{TMA}}\right)= & \operatorname{threshold}\left(\mathrm{CL}_{\mathrm{APM}}, u_{1}\right) \\
& +\operatorname{threshold}\left(\mathrm{CL}_{\mathrm{AS}}, u_{2}\right) \\
& +\operatorname{threshold}\left(\mathrm{CL}_{\mathrm{TMA}}, u_{3}\right),
\end{aligned}
$$

where $\mathrm{CL}_{\mathrm{APM}}, \mathrm{CL}_{\mathrm{AS}}$, and $\mathrm{CL}_{\mathrm{TMA}}$ are the confidence levels, and $u_{1}, u_{2}$, and $u_{3}$ are the mapping parameters. Majority voting is implemented by the rule: the best (based on CL) 2 methods out of 3 and adjust the mapping function for the 2 selected methods to the CL analogue to the adjusted mapping function in Eq. (28).

\section{Experimental Results}

For IRST, two distinct relevant operational scenarios exist. These two, the cold and the warm water environments, determine the main limitations of the system. We have chosen to perform experiments in these two scenarios.

\subsection{WWTs}

During the preliminary WWTs, the objective was to get insight into the passive ranging performance of the IRST sensor, since this was the first opportunity for the IRST sensor to be placed on a ship. The dynamical TMA algorithm used during the WWTs is described in Sec. 2.2.3. The knowledge of the absolute own sensor position on a moving platform at any time enabled the use of the full TMA algorithm.

On September 16, 2003, about 18 runs with typical passive ranging scenarios at different ranges to a target ship were performed. Typical scenarios were simulated as trial preparation, with added Gaussian bearing and elevation noise with a standard deviation of $1 \mathrm{mrad}$. All results presented are nonconstrained 6 degrees of freedom solutions (3-D position and velocity). This means that no a priori information was added to the TMA filter. Figure 6 illustrates a typical TMA simulation scenario used as trial preparation.

As explained in Sec. 2.2.3, TMA for dynamic targets requires a nonconstant course of the own ship. As trail preparation, a snake pattern was simulated. The TMA algorithm performed as expected, and the state vector solution stabilized at the simulated target.

\subsubsection{Results of the WWT scenario}

Figures 7 and 8 show the result of a 60-deg own ship pattern, starting at a range of $11.5 \mathrm{~km}$.

The own ship position in Fig. 7 is determined with global positioning satellite (GPS) data, and the reference target 


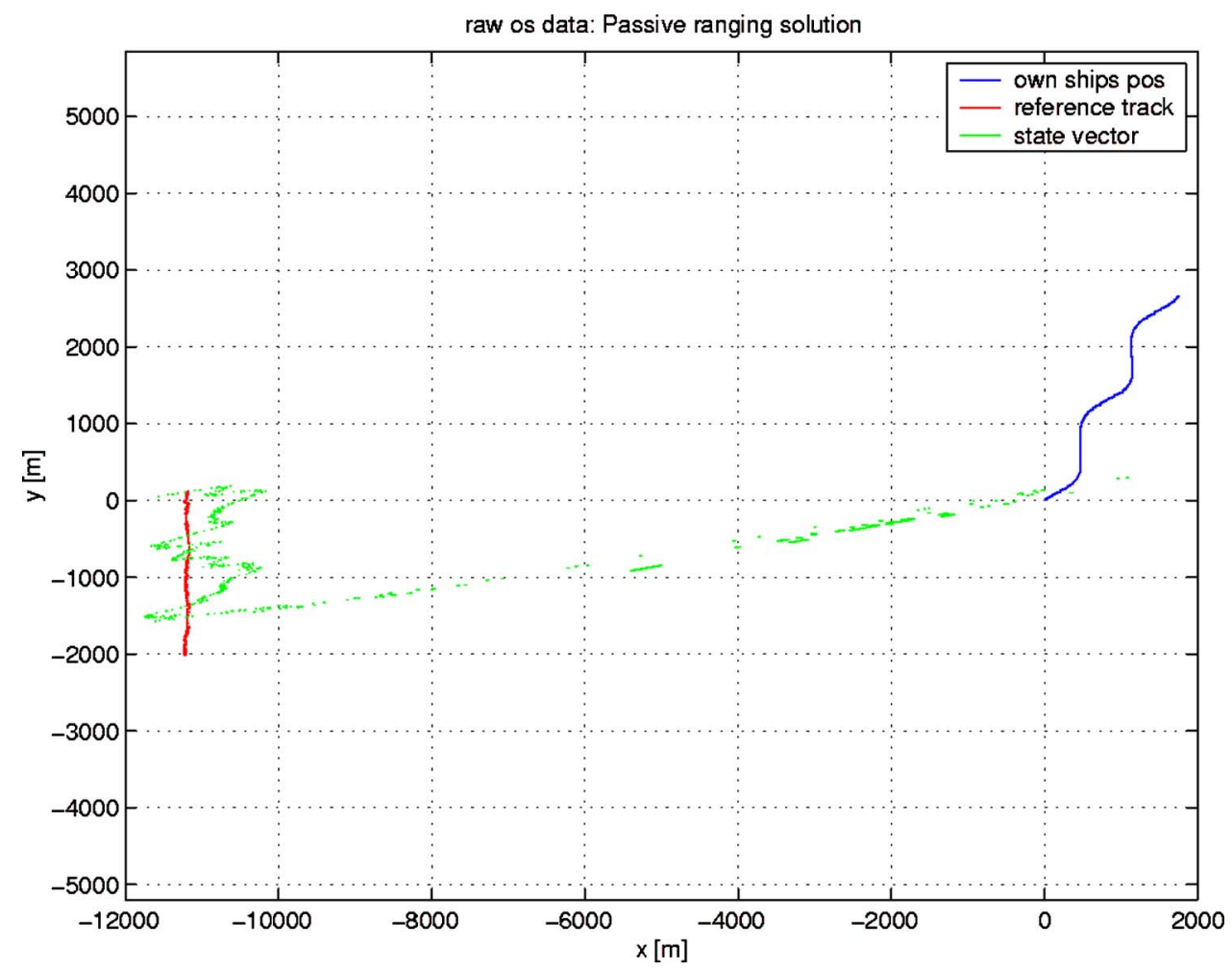

Fig. 7 Estimation of the state vector of a surface target; results of 60-deg own ships pattern, starting at a range of $11.5 \mathrm{~km}$.

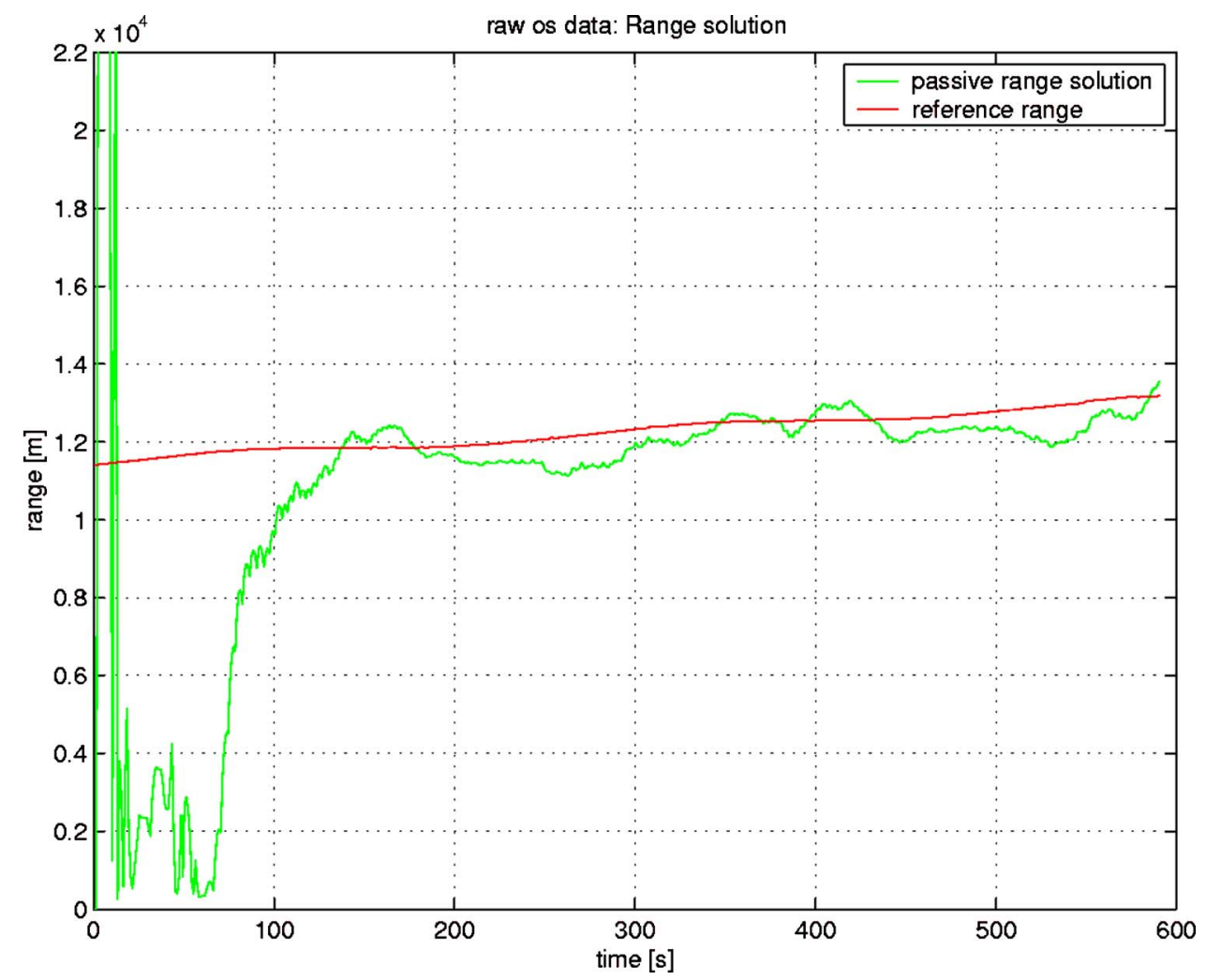

Fig. 8 Range estimation compared to reference range of the surface target; result of 60-deg own ships pattern, starting at a range of $11.5 \mathrm{~km}$. 
track is determined with the calibrated radar system of the own ship. Figure 8 explicitly shows the range solution of the state vector solution. Stabilization of the range solution is acquired after $100 \mathrm{~s}$.

\subsubsection{Analysis of the results}

Typical range accuracies of $10 \%$ were observed after run-in of the batch filters. Run-in is reached a few seconds after the first own ship maneuver. However, due to the required own ship maneuver, TMA for dynamic targets gives a good distance solution after $100 \mathrm{~s}$. The speed and course solutions look less stable, but improvement is possible when using a separate low-pass filter for velocity. Several parameters contribute to an accurate range solution. The shorttime stability of the own ship position (i.e., 3-D sensor position) has a large influence on the range solution. Small errors in the IRST sensor position result in larger ranging errors. The stability of the extended target track is important; a change in aspect angle with respect to the target can change the targets center of gravity, leading to biased observations. At short ranges, this effect is stronger than at long ranges.

\subsection{CWTS}

This subsection focuses on the scenario for the passive ranging test and the results during CWTs. The methods are implemented and applied to the CWT scenario data, and the results are evaluated with the actual ground truth distances measured with a radar system. The dynamical TMA method used in the WWT scenario performed well. For the CWT we used the stationary TMA version. The reason for choosing a less optimal variant of the TMA was the long time required (over $100 \mathrm{~s}$ ) for the "TMA dynamically variant" to come to a stable distance solution (see Fig. 8). Furthermore, the stationary location of the site and presence of head-on targets support this choice.

To apply stationary TMA, a simple straight-moving own ship is assumed. The input of the target information is based on the trials with the preproduction model of the dual-band IRST sensor at the LBTS location in Den Helder (NL). The runs were recorded in January 2003. The basic scenario is put in Cartesian coordinates. The basic 2-D scenario is shown in Fig. 9. The airplane began its inbound run at about $25 \mathrm{~km}$. Bearing information for the simulated ship is updated based on the true scenario data including ground truth range recordings and triangulation calculations:

new _ bearing $\Rightarrow \frac{\cos B_{\text {new }}}{\sin B_{\text {new }}}=\frac{y\left(t_{-} \text {new }\right)-v \Delta t}{x\left(t_{-} \text {new }\right)}$,

where $B_{\text {new }}$ is the new bearing, $x\left(t_{-}\right.$new $)$and $y\left(t_{-}\right.$new $)$are the $x$ and $y$ coordinates at the next time step, $v$ is the speed of the ship, and $\Delta t$ is the difference between time $t$ and $t_{-}$new.

The airplane trajectory is put in the basic scenario using radar system recordings. The preceding basic scenario serves well as a reference model. We use the bearing information and simulated bearing information from the basic scenario as input for the TMA distance estimation method and we test the TMA method on the same scenario. This is

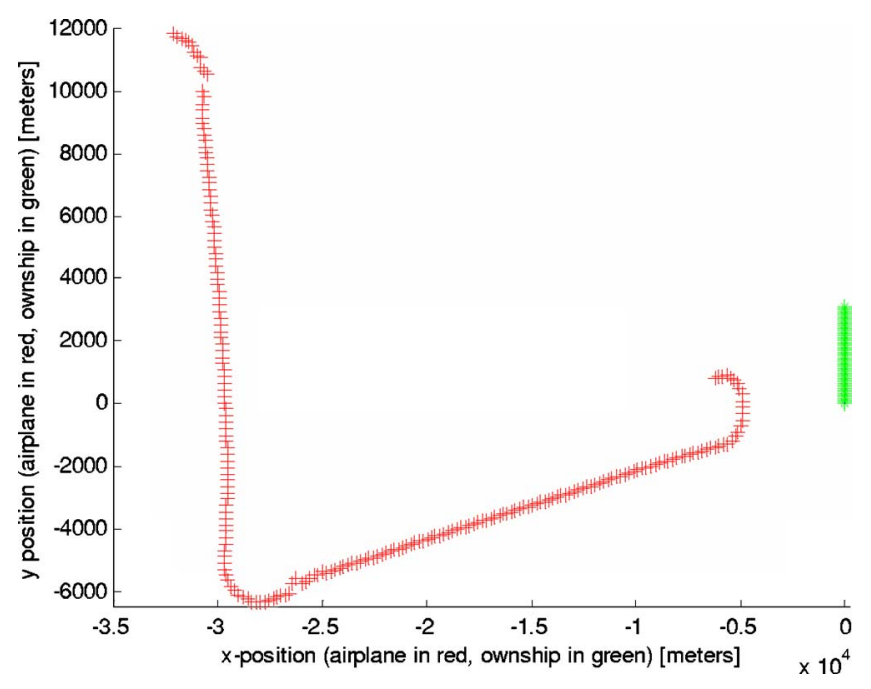

Fig. 9 Basic 2-D model with simulated own ship (green points) and airplane (red points) recordings at LBTS.

possible because the true range is removed from Eq. (19) and has no further impact on the bearing. Furthermore, uncertainty is added to the bearing.

\subsubsection{Results of the CWTs scenario}

The scenario just described is used to test the three passive ranging methods and the combined fusion methods. Results are displayed from the time stamp $t=150$.

APM. Results of the distance estimation APM method are presented in Fig. 10. The APM method is explained in Sec. 2.2.1. The distance estimate is obtained by combining the transmission estimates from IRBLEM, the calculated intensity from Eq. (1), and the estimated intrinsic radiation in Eq. (2). The distance uncertainty is calculated according to Eq. (23). The figure shows the distance and errors compared to the radar reference data. From the figure, we observe a good comparison between APM range estimates

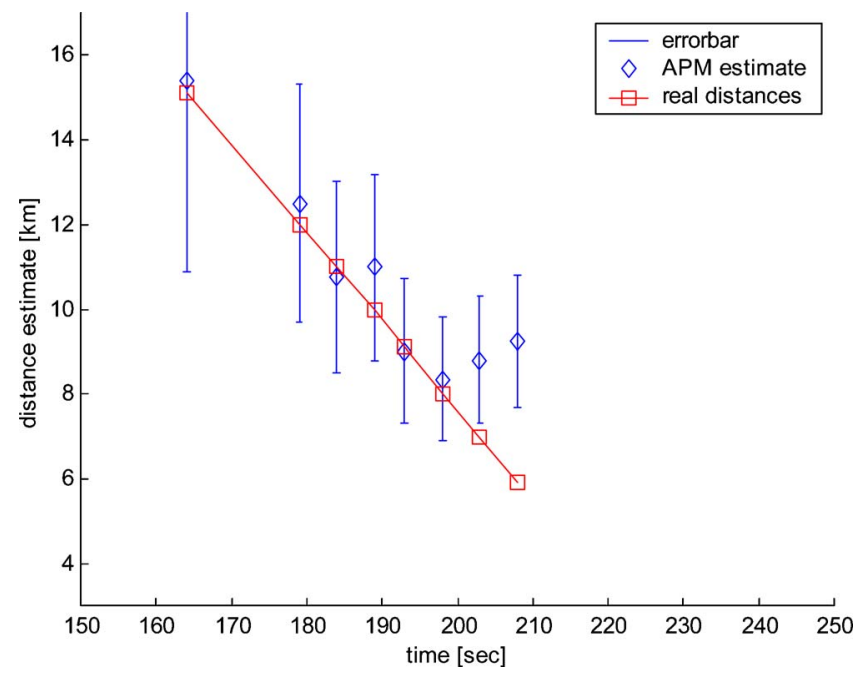

Fig. 10 APM distance and accuracy estimate compared to the reference data. 


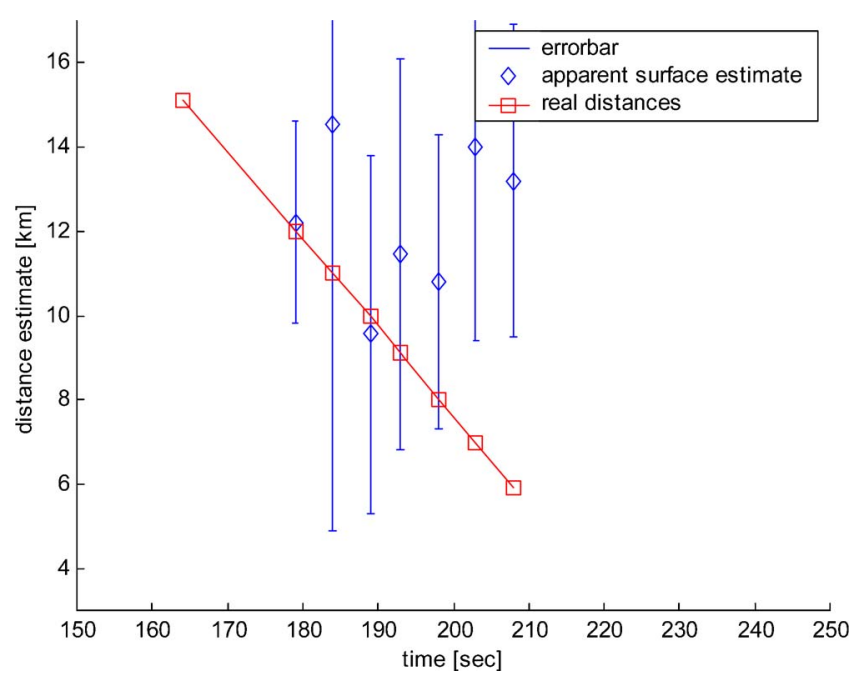

Fig. 11 AS distance and accuracy estimate compared to the reference data.

and the reference data, with some larger deviations at the short-range side of the graph and hence APM performs well and is stable.

AS. The AS of the target is obtained by counting all pixels above the peak value of the maximum of the background noise:

pixels $_{\text {target }}>$ max $_{\text {background }}$,

where pixels $s_{\text {target }}$ are the pixels belonging to the target and $\max _{\text {background }}$ is the maximum pixel value per image of the background. This rule is chosen because of stability reasons; fluctuations in the background noise are eliminated by this rule. The results are displayed for the LW band, as this is the dominating IR band in the cold scenario. Results are depicted in Fig. 11. The figure shows the distance en errors compared to the radar reference data. The AS method is explained in Sec. 2.2.2. The (change in) target area is retrieved form the available images. The changes in distance are available from the TMA process. Equation (5) then gives the distance estimate. Uncertainty calculations are performed analogously to those for the APM method. From the figure we conclude that the performance of this method for passive ranging at these ranges is not good.

TMA. Figure 12 shows the TMA results. The figure shows the distance and errors compared to the radar reference data. TMA for stationary targets is explained in Sec. 2.2.3. Bearing 1 is retrieved from the available information. Bearing 2 is calculated from the basic scenario in Sec. 4.1. The speed of the own ship is known. The difference in time is the time between the successive time steps. TMA distance estimates are calculated according to Eq. (19). Uncertainty calculations are performed analogously to those for the APM method. On average, the performance of the TMA

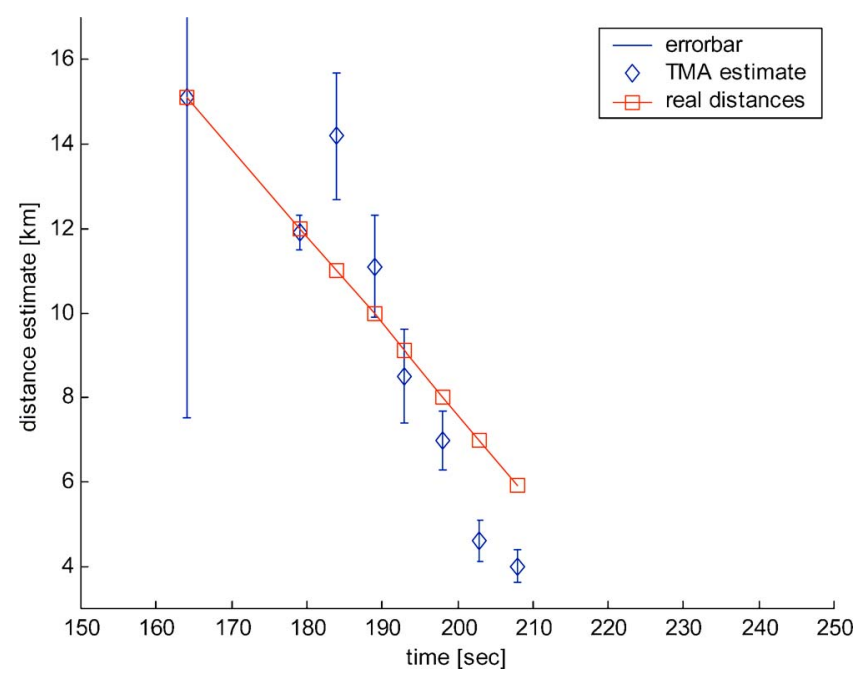

Fig. 12 TMA distance and accuracy estimate compared to the reference data.

method is fair. For the first measurement at $t=164$, the estimate is good but the uncertainty is very high. At a closer range the performance degrades.

Fusion methods. Before applying the fusion methods, the confidence levels are calculated according to Eq. (25). The confidence levels per time step, which are input for the fusion process, for each method are given in Table 3. The table shows that the TMA confidence levels are on average 0.04 higher than those for the APM (discarding the first time stamp). The AS confidence levels are always the lowest. Only at time stamps 164 and 193 is the confidence level higher for the APM method.

With the confidence levels from Table 3 and the fusion methods from Sec. 3.3, the distance estimations per fusion method are calculated.

To compare the performance of the separate distance estimation methods with the fusion methods the errors in

Table 3 Confidence levels per time step in CWTs.

\begin{tabular}{llll}
\hline \hline Time $(\mathrm{s})$ & $\mathrm{CL}_{\mathrm{APM}}$ & $\mathrm{CL}_{\text {AS }}$ & $\mathrm{CL}_{\mathrm{TMA}}$ \\
\hline 164 & $0.79^{\mathrm{a}}$ & 0 & 0.50 \\
179 & 0.85 & 0 & 0.97 \\
184 & 0.86 & 0.43 & 0.90 \\
189 & 0.86 & 0.67 & 0.90 \\
193 & $0.87^{\mathrm{a}}$ & 0.71 & 0.87 \\
198 & 0.88 & 0.79 & 0.90 \\
203 & 0.88 & 0.79 & 0.90 \\
208 & 0.89 & 0.83 & 0.90 \\
\hline \hline
\end{tabular}

${ }^{\mathrm{a} A C L}$ value is higher than TMA CL value at that range. 


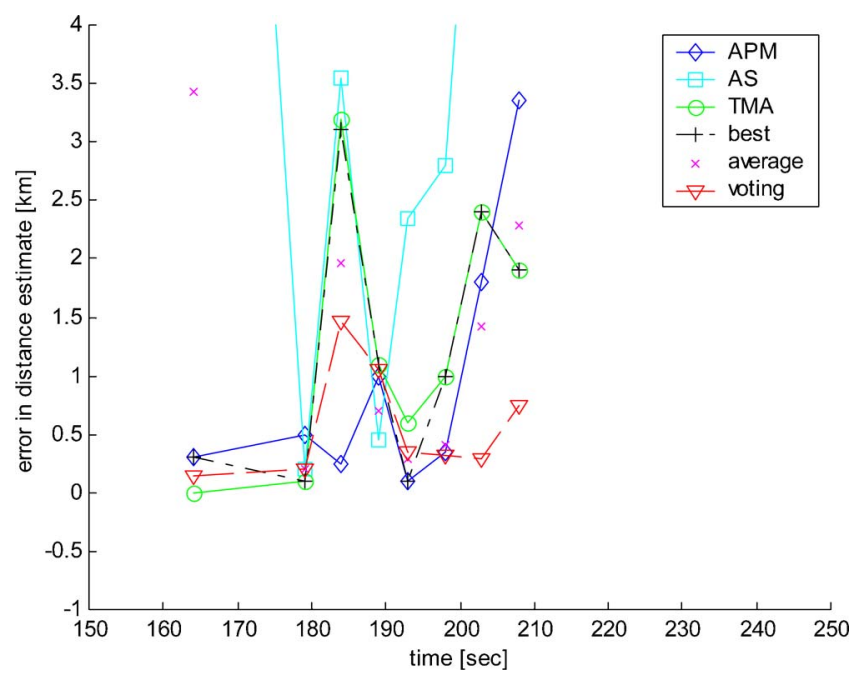

Fig. 13 Comparison of ground truth and the range estimates of the methods.

distance per method compared to the ground truth were calculated and are shown in Fig. 13. In Fig. 13 we see that at a distance of $8 \mathrm{~km}$, the error is within an accuracy of about $5 \%$ for the fusion method "majority voting" and the APM method. Results for closer ranges of the target are not within this accuracy.

The mean and standard deviation of these errors of the three separate methods and three fusion methods compared to ground truth are given in Table 4 . The mean error $\left(\mu_{\text {error }}\right)$ is calculated according to

$\mu_{\text {error }}=\frac{1}{N} \sum_{t=1}^{N}\left|R_{\text {predicted }}(t)-R_{\text {ground_truth }}(t)\right|$,

where $N$ is the number of measurements, $R_{\text {predicted }}$ is the predicted range, and $R_{\text {ground_truth }}$ is the ground truth range at time $t$. The standard deviation of the error $\left(\sigma_{\text {error }}\right)$ is also given, which is the standard deviation of the mean error. Furthermore, the mean of the uncertainty $\left(\mu_{-} \Delta R\right)$ per method, with $\Delta R$ described in Sec. 3.2, is given in Table 4 according to

Table 4 Mean, standard deviation of the error, and the mean uncertainty comparison between the methods in CWTs.

\begin{tabular}{lllllll}
\hline \hline Method Error & APM & AS & TMA & Best & Average & Maj. Voting \\
\hline$\mu_{\text {error }}$ & 0.96 & 4.84 & 1.29 & 1.25 & 1.34 & 0.57 \\
$\sigma_{\text {error }}$ & 1.11 & 4.91 & 1.13 & 1.12 & 1.15 & 0.47 \\
$\mu \_\Delta R$ & 2.24 & 4.09 & 1.68 & 1.36 & 2.46 & 1.90 \\
\hline \hline
\end{tabular}

$\mu_{-} \Delta R=\frac{1}{N} \sum_{t=1}^{N} \Delta R(t)$

where $N$ is the number of measurements, and $\Delta R$ is the uncertainty at time $t$. From Table 4 we note that the average of our a priori estimate for the distance uncertainties compares well with the measured errors for the methods AS, TMA, and Best (0.8 to 1.3 times). However, our a priori estimate apparently was higher than the measured errors for methods APM (2.3 times), average (1.8), and majority voting (3.3). The majority voting method has the lowest mean error, $41 \%$ less than the APM method.

\subsubsection{Analysis of the results}

In this section we analyze the results of the CWT by comparing the results of the different passive ranging approaches.

The APM method performs well and is stable. The performance degradation at closer distances is caused by "clipping" (reaching the maximum value in the digital image) of the peak value of the intensity of the target. The main reason for the large uncertainty range in this method is the uncertainty in the estimated target radiant intensity $W$ in Eq. (2). Based on Table 4 the APM method has the best evaluated error of the three methods APM, AS, and TMA.

The AS method performs poorly in these ranges. This method is very sensitive to initial input parameters. The reason for the poor results of this method is the fluctuation in the values of the target intensity in the images, which results in poor target size estimates. Results should improve when higher resolution images are available.

The TMA method gives fair results, and with lower uncertainty than the other separate methods. The described method for TMA does not work for a so-called weaving target, which was the case during the beginning and the end of the track trial. When a target is at a steady course and is coming inbound to the ship, the method gives a distance indication. Furthermore, the time between distance estimations is chosen arbitrarily here. This gives more uncertainty when the time between two distance estimations is small (for example, for the first measurement at $t=164$ ). The performance degradation at closer ranges is due to the sensitivity for the errors in the model (at that moment, the target is no longer stationary).

The establishment of confidence levels from range uncertainties seems good. When the target approaches, the confidence levels are higher. This is correct because at closer ranges more information is gathered and the uncertainty in the distance estimate is smaller. There is, however, no influence of the (non-) correctness of the distance estimation on the confidence level. This requires further research. Furthermore, as we have seen that the uncertainty for the APM method was substantially larger than the finally recorded errors, fusion approaches that are now dominated by the TMA, may be improved when the uncertainties can be decreased in the other methods.

The fusion method of majority voting shows an improvement in the distance estimation error of $41 \%$ in comparison (see Table 4) with the best nonfused method (APM). Because only one scenario is tested, this result can- 
not necessarily be extended to more scenarios. The weighted average fusion method performs worse than APM and TMA because of the influence of the AS method. The best method performs worse than APM because of the influence of TMA and its high confidence levels.

\section{Discussion}

The APM method performs well and is stable. Improvement of the estimation at closer distances would be obtained by extrapolating the clipped values of the peak intensity of the target to imaginary higher values. The uncertainty in the distance estimation is reduced if the uncertainty for the input parameter $W$ (target radiant intensity) can be reduced. The fact that the observed error is substantially lower than the a priori uncertainty gives some information that this method may require more optimization. A disadvantage of this method is that the transmission in the direction of the target and the target signature are required input data. Besides that, background intensity and standard deviation of the IRST image must also be calculated at every time step. Calculation of the TTG is possible with this method. This is used for priority scaling of targets in the IRST system.

Typical antisurface warfare scenarios were performed during WWTs. Bearing and elevation resolution of the imaging system has a large influence on the performance of the TMA algorithm. This is, however, a cost driver and, in most cases, a given property. Future investigation should also include how other types of information, as discussed in this paper, could be fused in the TMA algorithm. In this way, more stressing anti-air warfare scenarios could also benefit from passive ranging techniques. The poor performance of the AS method might well be improved by using more sensitive IR equipment with higher spatial resolution. Additionally, image analysis tools, such as, for instance, dynamic superresolution, are essential to apply this technique with good range performance and low uncertainties.

The TMA stationary method should perform better when it is applied with the use of track filters, as is shown during the WWTs. Especially, predictions from a filter for positions of the target in the near future are useful for the distance estimation. During the CWT test scenario the updated $x$ and $y$ positions of the target are calculated with the reference distance to the target. This was done to demonstrate the principle of the TMA stationary method. A simulated scenario would give the same information for the TMA stationary variant. The live test scenario mainly added extra information for the possible fusion of the APM and AS distance estimation methods. Furthermore, tests with the preproduction model and live bearing data placed aboard an ADCF have not yet been performed but are a subject for further research.

Little information is found in literature about fusion of passive ranging methods. Our investigation to combine different passive ranging methods looks promising. The fusion method majority voting shows a better performance than the separate APM and TMA methods. A better performance can be achieved by enhancing the confidence levels. The influence of the error for each method compared to ground truth can improve this confidence level. The uncertainty in the methods (and confidence levels) is probably reduced when correlation between the input parameters is imple- mented in the overall uncertainty analysis. The input parameter sensitivity analysis performed here assumed independent input parameters. Additionally, the APM and AS methods require ranging guidance from some external source for the initial positioning. In our case, the TMA value was used. This also shows that a passive ranging fusion system is required, and that a priori uncertainty estimates may be correlated between the various methods.

Future work should focus on the method "intensitydistance diagrams," which are only briefly mentioned in Sec. 2. Using databases with targets and their IR transmissions in the MWIR and LWIR bands, will optimize the dual-band opportunities of the sensor in question. Practical future work is possible when the sensor is placed aboard the ADCF, the implementation of a software module for "distance indications" provides improved situational awareness. An extension for the distance estimation is possible when the bearing information of the IRST sensor is fused with the bearing information from the trainable electrooptical observation system (TEOOS) that is already placed aboard the ADCF (TEOOS is also equipped with a laser range finder).

Passive ranging is an elegant method to acquire a 3-D recognized air and surface picture of the near surroundings of a naval ship without giving away the own ship position. Furthermore, imaging sensor systems give additional visual information to the operator in the command center, information that is more important today during brown water operations.

\section{Conclusions}

We discussed three separate methods for passive ranging with an IRST sensor and two practical implementations with real data. The TMA algorithm in combination with the IRST system, used during WWTs, proved to give good results in real antisurface warfare scenarios. Accuracies of 10 were obtained. For anti-air warfare scenarios, where reaction time is a larger issue, other types of information fusion are still required and are a subject for further investigation.

We demonstrated passive ranging capability of an IR sensor, with a certain level of accuracy. Based on the CWT scenario and the passive ranging methods atmospheric propagation, AS of the target, stationary TMA, and the fusion of these methods, we demonstrated that fusion of information improves the distance estimation for a realistic scenario. The fusion method majority voting improves the distance estimation with $41 \%$ compared to the best separate APM method. This best separate method already performs quite well and is stable. An accuracy of the distance estimation of $5 \%$ at $8 \mathrm{~km}$ was reached for the specific test scenario. The results of the AS of the target method are in general not good. A recommendation for future work is to perform tests at an Air Defense Command Frigate when the IRST sensor is placed aboard the ship.

\section{Acknowledgments}

The RNLN allowed the main author to spend time conducting this research besides his normal duties. The manufacturer of the IRST sensor, Thales Naval Netherlands, has provided the reference data. 


\section{References}

1. R. Knepper, "Sirius, a long range infra red search and track system," in Infrared Technology and Applications, Proc. SPIE 3061, 578-584 (1997).

2. S. S. Blackman and R. Popoli, "Passive sensor tracking methods," and "Multiple sensor tracking," Chaps. 5 and 10 in Design and Analysis of Modern Tracking Systems, pp. 259-324, 661-736, Artech House, Norwood, MA (1999).

3. R. van Sweeden, H. M. A. Schleijpen, and P. B. W. Schwering, "Passive ranging techniques," EUCLID RTP 8.2, START WE.256.4F, The Hague, The Netherlands (1996).

4. J. F. de Groot, "Tracking aspects of SIRIUS (additional) functionality," Internal Document, Thales Naval Netherlands, Hengelo, The Netherlands (1996).

5. R. A. W. Kemp, "Point target detection, tracking and passive ranging: a performance analysis study," Chap. 19, TNO report FEL-99-S230, The Hague, The Netherlands (1999)

6. P. B. W. Schwering, A. N. de Jong, and J. Winkel, "Eindrapport SIRPS (Staring Infrared Panoramic Sensor)," TNO report FEL-01A309 (in Dutch), The Hague, The Netherlands (2004).

7. T. Aytaç and B. Barshan, "Rule-based target differentiation and position estimation based on infrared intensity measurements," Opt. Eng. 42(6), 1766-1771 (2003).

8. O. Sasaki, K. Sakata, and T. Suzuki, "Optical method for detecting displacement of a car in stereo images," Opt. Eng. 42(7), 2092-2095 (2003).

9. S. Ono and S. Komatsu, "Simultaneous image restoration and distance estimation of an object from a strongly defocused photon image," Opt. Eng. 42(4), 1024-1028 (2003).

10. Ruters and Michalowicz, "Passive location and ranging," in The IR\&EO Systems Handbook, Vol. 5, S. B. Campana, Ed., Chap. 4.5, pp. 336-341 (1993).

11. A. N. de Jong and H. Winkel, "Enhanced IR point target detection by atmospheric effects," in Infrared Technology and Applications XXVIII, Proc. SPIE 4820, 885-896 (2002).

12. A. N. de Jong and H. Winkel, "Report on the Baltic 99 IR experiments," TNO report FEL-00-A094, The Hague, The Netherlands (2000).

13. A. N. de Jong, J. Winkel, M. M. Moerman, and R. de Rooy, "Preliminary report on the TG16 POLLEX trial," TNO-report FEL-01A222, The Hague, The Netherlands (Sep. 2001).

14. A. N. de Jong, H. Winkel, M. Moerman, K. Stein, K. Weis-Wrana, L. Forand, G. Potvin, J. Buss, A. Cini, H. Vogel, and A. Stark, "TG16 Point target detection experiment POLLEX," in Infrared Technology and applications XXVIII, Proc. SPIE 4820, 849-860 (2002).

15. D. Dion, L. Gardenal, L. Forand, M. Duffy, G. Potvin, and S. Daigle, "IR boundary layer effects model (IRBLEM) — version 4.1," DRDCValcartier, Canada (Dec. 2002).

16. S. S. Blackman and R. Popoli, "Infrared search and track systems," Sec. 2.3 in Design and Analysis of Modern Tracking Systems, pp. 85-119, Artech House, Norwood, MA (1999).

17. S. S. Blackman and R. Popoli, "Passive sensor tracking methods," Chap. 5 in Design and Analysis of Modern Tracking Systems, pp. 268-271, Artech House, Norwood, MA (1999).

18. U.S. Navy, Navy Combat Manuals, "Operations specialist, volume 1," Chap. 10, pp. 10-23, U.S. Navy (1999).

19. F. Cremer, K. Schutte, J. G. M. Schavemaker, and E. den Breejen, "A comparison of decision-level sensor-fusion methods for antipersonnel landmine detection," Inf. Fusion 2, 187-208 (2001).

20. J. G. M. Schavemaker, E. den Breejen, F. Cremer, K. Schutte, and K. W. Benoist, "Depth fusion for anti-personnel landmine detection," in Detection and Remediation Technologies for Mines and Minelike Target VI, Proc. SPIE 4394, 1071-1081 (2001).
Maarten de Visser attended the Royal Netherlands Naval Academy and graduated in 1999 in situational awareness and refinement. He received his MSc degree from the electrical engineering faculty of Delft University of Technology in 2004. His research was the subject of this paper. After several years serving as deputy weapons officer aboard different types of frigates, he is currently working in the field of project engineering for the Royal Netherlands Navy (RNLN). His interests are in the practical applications and implementations of enhancing situational awareness aboard frigates.

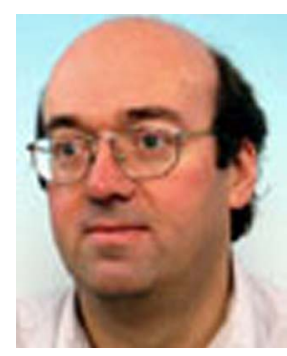

Piet B. W. Schwering received his MSc degree in astronomy in 1983 and his PhD degree in mathematical sciences in 1988, both from the University of Leiden, where from 1983 to 1987 he was working in the field of astrophysics, and he studied dust properties and star formation in the Magellanic clouds using Infrared Astronomical Satellite (IRAS) data at IR wavelengths. Since 1987 he has been a scientist and project manager in the Electro-Optics Group, the Netherlands Organization for Applied Scientific Research (TNO). His interests are the detection of objects in IR surveillance, the characteristics and reduction of background clutter, and sensor system evaluation.

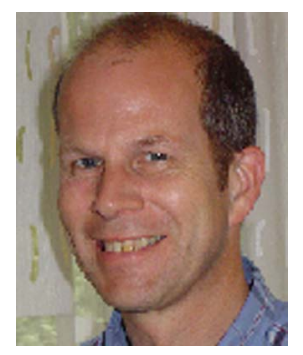

Johannes F. de Groot received his MSc degree from the electrical engineering faculty of Delft University of Technology in 1982 and joined Thales Hengelo, the former Hollandse Signaalapparaten. His main involvement was in the development and evaluation of some generations of longrange infrared search and track systems (LRIRST). His main interest is signal and data processing, with a special interest in passive ranging by means of target motion analysis (TMA). He is currently working on algorithm development and prototyping of next-generation staring IRST systems.

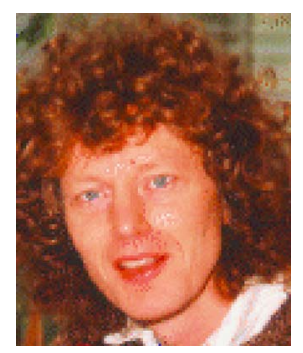

Emile A. Hendriks received his MSc and $\mathrm{PhD}$ degrees, both in physics, from the University of Utrecht in 1983 and 1987, respectively, and he became an assistant professor with the electrical engineering faculty of the Delft University of Technology. In 1994 he became a staff member of the Information and Communication Theory of this faculty, and since 1997 he has headed the computer vision section of this group as an associate professor. His interests are computer vision, low-level image processing, image segmentation, stereoscopic and 3-D imaging, motion and disparity estimation, structure from motion/disparity/silhouette, and real-time algorithms for computer vision applications. 